\title{
Enrichment and Separation of Methane Gas by Vacuum Pressure Swing Adsorption
}

\author{
Tao Zhu ${ }^{(D,},{ }^{1,2,3}$ Ruonan Wang, ${ }^{1}$ Xing Zhang, ${ }^{1}$ Yiwei Han, ${ }^{1}$ Wenjing Bian, ${ }^{1}$ Yan Ma, \\ and Ming Xue ${ }^{2}$ \\ ${ }^{1}$ School of Chemical \& Environmental Engineering, China University of Mining \& Technology (Beijing), Beijing 100083, China \\ ${ }^{2}$ State Key Laboratory of Petroleum Pollution Control, Beijing 102206, China \\ ${ }^{3}$ Shanxi Key Laboratory of Lacustrine Shale Gas Accumulation and Exploitation (under planning), Shanxi Province, China
}

Correspondence should be addressed to Tao Zhu; bamboozt@cumtb.edu.cn

Received 14 June 2020; Accepted 8 March 2021; Published 9 April 2021

Academic Editor: Fateme Rezaei

Copyright (C) 2021 Tao Zhu et al. This is an open access article distributed under the Creative Commons Attribution License, which permits unrestricted use, distribution, and reproduction in any medium, provided the original work is properly cited.

\begin{abstract}
In China, owing to the methane concentration being below $0.75 \%$, the coal ventilation air methane (CVAM) is usually emitted directly into the atmosphere, rather than utilized, which not only causes huge waste of energy but also exerts potential hazards to the greenhouse effect. It is important and practicable to save costs of development and investment by simulating enrichment and separation of CVAM with an aim to improve the efficiency and recovery of adsorption separation. Above all, it will have important practical significance to the development of adsorption separation. In this paper, the experiment of the pressure swing adsorption process was carried out on double towers built by our laboratory, and the Aspen Adsorption was used to simulate the process. The effect of the operation parameters on the desorbed methane concentration was studied by altering the feed concentration, the adsorbed pressure, and adsorbed and desorbed time. The results of simulation and experiment are basically consistent. The ratio of methane was decreased following the increasing concentration of the feed. The optimum adsorption pressure and time were found to be $210 \mathrm{kPa}$ and $120 \mathrm{~s}$, respectively. The optimum desorption times of experiment and simulation were $150 \mathrm{~s}$ and $120 \mathrm{~s}$, respectively. Because there was a man-made $30 \mathrm{~s}$ time lag between the experiment and simulation to protect the vacuum pump, the results show that the simulation and experiment were matched well. Therefore, we can make use of Aspen Adsorption to design separation and enrichment of CVAM, providing theoretical and practical guidance for the gas separation and saving resources and energy.
\end{abstract}

\section{Introduction}

Enrichment and separation of methane by PSA is a dynamic process. The parameters of the system, such as pressure, concentration, and temperature, are difficult to measure due to the complexity and variability. At the same time, if the requirements of products and environmental conditions are different, the corresponding pressure swing adsorption devices and processes will be different [1-4]. The simulation study of pressure swing adsorption can reduce the cost of research and development, reduce the cost of investment and energy consumption, and improve the efficiency of adsorption-separation and recovery. It has importantly practical significance for the development of adsorptionseparation technology.
Kumar et al. [5] carried out the simulation study of pressure swing adsorption by SIMPAC software, which provided a number of adsorption equilibrium isotherms. Liu and Ritter [6] considered the whole process of four-step pressure swing adsorption to simulate solvent recovery. In their simulation process, although nonisothermal and nonadiabatic treatment conditions were adopted, the change of longitudinal pressure drop in the adsorption tower was ignored. Jiang et al. [7] simulated the air separation oxygen production process on the basis of solving differential equations in the simulation model by independently designed solver. After comparing several different methods, Jiang et al. [7] concluded that finite volume can more accurately reflect the process of adsorption. In order to save calculation resources, he also used the direct determination method to shorten the 
calculation process of cyclic convergence during the calculation. Bárcia et al. [8] filled the adsorption tower with a certain proportion of $\mathrm{C} 5$ and $\mathrm{C} 6$ adsorbents. Under the conditions of nonisothermal and adiabatic, they simulated the dynamic process of PSA by using the Aspen Adsim software, and the simulation results showed that the software could reflect the experimental results well. Yang et al. [9] successfully simulated the process of separating and purifying $\mathrm{H}_{2}$ from the mixed gas by multibed PSA using the Aspen Adsim software. Based on the single-tower process, Ding and Levan [10] not only studied the direct determination method in cyclic steady-state limiting method but also saved the cost of convergence calculation to some extent. However, although this method saved the time of simulation to achieve steady state, it increased the difficulty of calculation and failed to describe the trend of state variables in detail. Jiang et al. [11] compared the effect of different partial differential solving methods on the accuracy of the results of PSA simulation and found that the finite volume method is more suitable for the simulation of VPSA process than the finite difference method and the finite element method. Although the research of Jiang et al. [11] accelerated the convergence of the model, the steady-state result obtained in the end was only an approximation, which could not meet the design requirements of industrial application. Nilchan and Pantelides [12] simulated the simple RPSA oxygen production process by discretizing the differential equations using the finite difference method. Although the full discretization method reduced the difficulty of solving the differential equation by transforming it into an algebraic equation, it increased the amount of calculation, which made it difficult to converge when simulating the complex PSA process. Silva et al. [13] developed a general package for simulation of fixed-bed and cyclic adsorption processes, and two cyclic adsorptive separations of propylene/propane system and $\mathrm{n}$ /iso paraffins mixtures had been tested, and experimental breakthrough data from both systems were compared with simulated results showing reasonable agreement. Yang et al. [14] developed a cost-effective activated carbon bead adsorption and successive $\mathrm{CO}_{2}$ displacement method for the recovery of ventilation air methane, where $10 \% \mathrm{CH}_{4}$ gas is concentrated to $89 \%$, and $1 \% \mathrm{CH}_{4}$ in VAM gas can be concentrated to $53.9 \%$. Qu et al. $[15,17]$ studied the effect of the microstructure of carbon on the $\mathrm{CH}_{4} / \mathrm{N}_{2}$ separation and $\mathrm{CH}_{4}$ enrichment from the low-concentration methane gas mixed with nitrogen and developed mathematical model with IAST-Sips formulation to simulate the enrichment of low-grade methane gas from nitrogen mixture by VPSA with $\mathrm{CO}_{2}$ displacement process, and the results showed that a feed gas with $1 \% \mathrm{CH}_{4}$ is enriched to $75.40 \%$ with $89.02 \%$ recovery by the proposed process.

Although the simulation of pressure swing adsorption has been applied in many fields, including nitrogen and methane separation, there has not been any simulation study on PSA technology to enrich and separate the extremely low concentration of ventilation air methane. At the same time, obtaining the best operating conditions of the detailed PSA bed model and detailed characteristics of the new adsorbent dynamics and adsorption equilibrium to accurately establish the multicomponent adsorption isotherm and mass transfer model is still a great challenge for existing and emerging applications to develop periodic systematic simulation of the multibed PSA. Therefore, on the basis of laboratorybuilt two-tower PSA device, this paper fitted related parameters on the operating platform with Aspen Adsorption and established PSA simulation model of enriching and separating ventilation air methane. The effects of feed gas concentration, pressure, adsorption time, and desorption time on methane concentration were investigated. In this paper, we only consider the methane and nitrogen in the CVAM, and there are other gases in the CVAM, which are beyond the scope of our study; so, they will not be considered. This study can provide data support for the enrichment and separation of ventilation air methane, which may reduce costs of development and investment, decrease energy consumption, and improve the efficiency of adsorption-separation and recovery. It also provides a reference for the explosion protection problem in the process of high concentration gas treatment in the future.

\section{Materials and Methods}

2.1. Experimental Device. The feed gas is a fully mixed gas of air and pure methane, which enters the adsorption tower filled with activated carbon from the bottom of the tower for adsorption through mass flowmeter and methane detector. The strong adsorption component (methane) is adsorbed by activated carbon and stays in the tower, while the weak adsorption components (nitrogen and oxygen) flow out from the top of the tower. The effluent gas is recovered after the methane concentration is measured by the methane detector. When the adsorption is complete, in order to prevent the vacuum from burning out, the vacuum pump should be opened for desorption after the pressure in the tower drops to a certain degree. The desorbed gas is relatively high concentration of methane, which is called desorption gas or product gas. The flow and methane concentration of feed gas, product gas, and effluent gas are monitored by mass flowmeter and methanometer, and the pressure change in the tower is monitored by a pressure sensor in real-time, and the data is imported into the computer through the self-designed circuit system. Specifically, the experimental device is composed of six important components: gas source system, adsorption-desorption system, control system, measurement system, and pipe connection system. The flow chart is shown in Figure 1.

The gas source system is mainly composed of methane cylinder, mass flowmeter, air compressor, buffer tank, drying tank, solenoid valve, and methanometer. The air compressor with an input power of $0.55 \mathrm{~kW}$ compresses the air into the buffer tank. After being evenly mixed with $99.9 \%$ pure methane gas, the mixed gas is sent to the adsorption system through the drying tank, and the output flow is controlled by the mass flowmeter. The air compressor is a type of ZW-50A piston compressor, and the air inflow is about $5 \mathrm{~L} / \mathrm{min}$. In this experiment, the feed gas was simulated by uniformly mixing pure methane and air, and the concentration varied from $0.1 \%$ to $0.7 \%$. 


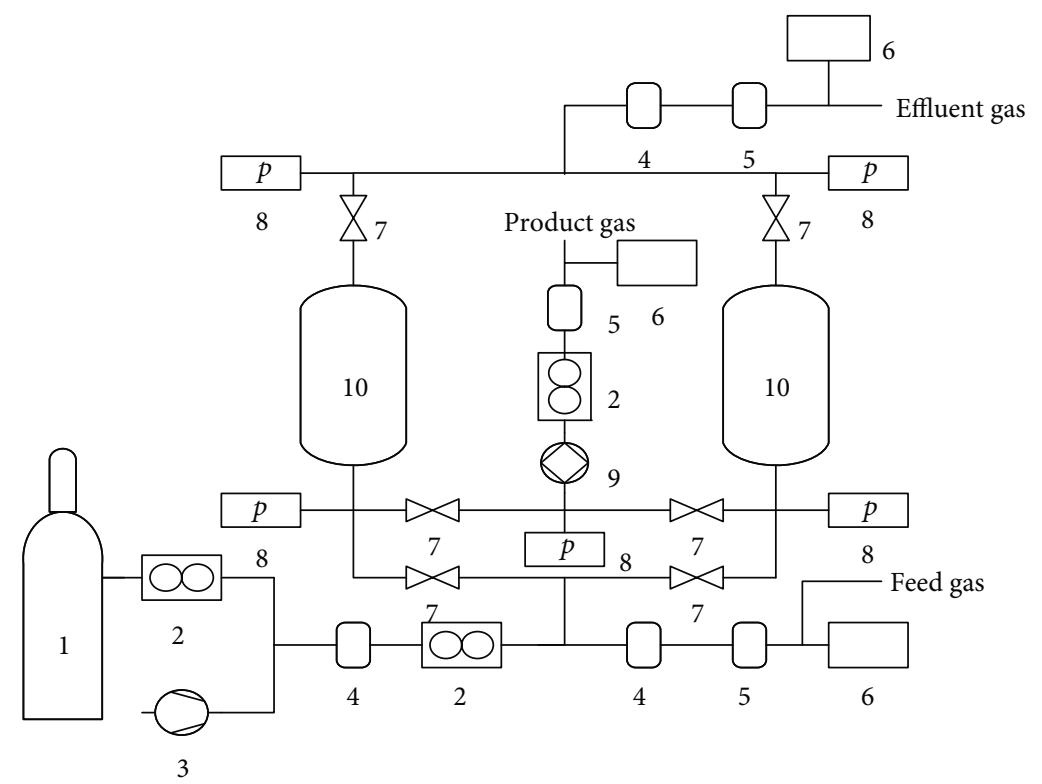

Figure 1: Experimental system: (1) methane cylinder, (2) mass flowmeter, (3) air compressor, (4) buffer tank, (5) drying tank, (6) methanometer, (7) solenoid valve, (8) pressure sensors, (9) vacuum pump, and (10) adsorption tower.

The adsorption-desorption system is composed of adsorption tower, vacuum pump, solenoid control, pressure sensors, and methanometer. Adsorption tower and vacuum pump are important components in the adsorptiondesorption system. The size and internal structure of the adsorption tower directly affect the flow rate, adsorption rate, and contact area with the adsorbent of the mixed gas. The power of the vacuum pump directly affects the desorption pressure, and within a certain range, the methane concentration varies with the desorption pressure.

The control system and measurement system mainly display readings of each instrument and then control the solenoid valve by the PLC module and import the data into the computer. The main function of the pipe connection system is to connect, support, and protect each part. The pipe connection system mainly includes clamp nozzle, connector, connecting pipe, three-way valve, three-hole quick-insert connector, and support seat.

2.2. Cyclic Process. According to the variation of system pressure, pressure swing adsorption can be divided into atmospheric adsorption and vacuum desorption, pressure adsorption and atmospheric desorption, and pressure adsorption and vacuum desorption. Pressure adsorption and vacuum desorption is called vacuum pressure swing adsorption (VPSA). This study adopted atmospheric pressure adsorption and vacuum desorption. Under atmospheric adsorption, the adsorption capacity of activated carbon for methane increases linearly with time within a certain time range. In the adsorption process, methane and other strong adsorptive components were adsorbed by activated carbon and remained in the adsorption tower, and weak adsorptive components such as nitrogen and oxygen were discharged from the upper end of the tower. In the desorption process, the vacuum pump vacuumized the adsorption tower so that the methane adsorbed by activated carbon was desorbed out, and the product gas with high volume fraction of methane was extracted from the bottom of the tower.

The specific operation of the two adsorption towers is shown in Figure 2. V-1, V-2, V-3, V-4, V-5, and V-6 are solenoid valves. $\mathrm{V}-1, \mathrm{~V}-3$, and $\mathrm{V}-5$ are used to control adsorption tower 1 , and V-2, V-4, and V-6 are used to control adsorption tower 2. Take tower 1 for example, the valves V-3 and V-5 were opened, and V-1 was closed, and the feed gas entered adsorption tower 1 from V-5and fully contacted with the adsorbent filled in the tower and was absorbed until penetration. The weak adsorptive components in the feed gas were discharged through V-3. After the adsorption was completed and the effluent gas was discharged, the V-3 and V-5 were closed, and V-1 was opened, and the tower 1 was vacuumed. The extracted gas was desorption gas with high methane concentration, which could be directly passed into the catalytic oxidation device for further treatment. In this study, the two adsorption towers work independently at the same time, and both go through four stages of intake, adsorption, depressurization, and desorption. The specific process is shown in Figure 3.

2.3. Adsorbent. The adsorbent is the core of pressure swing adsorption experiment. The property of the adsorbent directly affects the concentration of methane in product gas. The absorbent used in this study was activated carbon made by ourselves. The specific surface area, pore volume, and pore diameter of the adsorbent were measured by the liquid nitrogen adsorption method in this study. Breakthrough curve and saturated adsorption capacity of nitrogen and methane in the activated carbon adsorption bed were determined by pressure swing adsorption device. The performance of the adsorbent was verified by half cycle experiment. This study characterized the activated carbon and estimated the balance parameters and kinetic parameters of nitrogen and methane. The twin tower pressure swing 


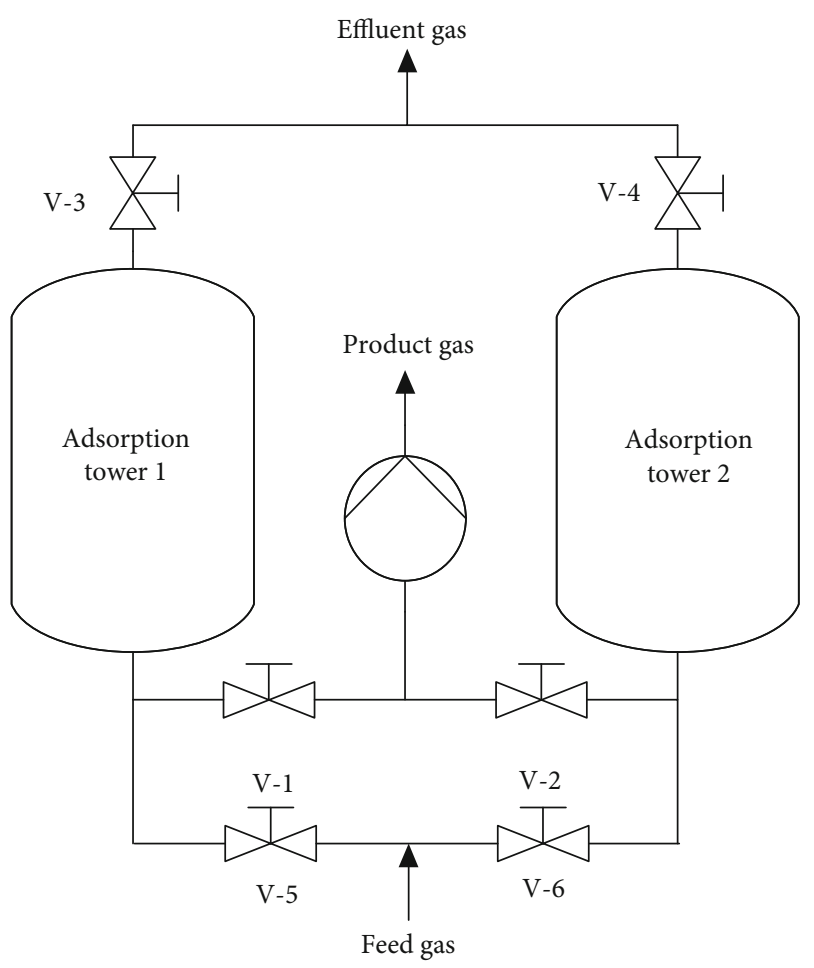

Figure 2: Flow chart of pressure swing adsorption tower.
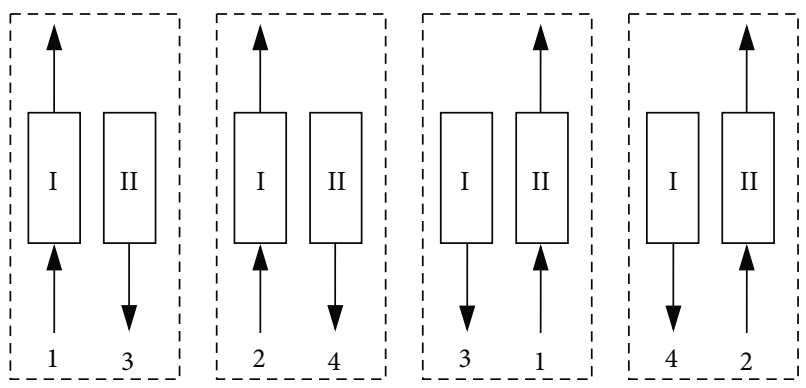

FIGURE 3: Schematic diagram of the PSA process of adsorption tower 1 and 2: (1) intake, (2) adsorption, (3) depressurization, and (4) desorption. (I) Adsorption tower 1. (II) Adsorption tower 2.

adsorption experiment device was adopted to test the separation process of nitrogen and methane.

In this paper, the adsorption and desorption isotherm curves of the activated carbon were measured by the JWBK static nitrogen adsorption instrument, and the parameters such as specific surface area, pore volume, and saturated adsorption capacity were calculated and listed in Table 1.

\subsection{Pressure Swing Adsorption Simulation}

2.4.1. Pressure Swing Adsorption Simulation Module of Aspen Adsorption. The pressure swing adsorption simulation module of Aspen Adsorption is shown in Figure 4. The core of the simulation is the settings of adsorption tower and the PSA process. By initializing each module in the flow chart and then using the cycle controller to set the steps of the process, the PSA could be simulated dynamically [16].
2.4.2. Simulation Environment Settings. Although the adsorption process is an exothermic process, the heat released by the adsorption will not significantly affect the bed temperature because methane is a trace amount. Therefore, the whole process was set to be isothermal. The isotherm is an extended Langmuir isotherm, and the form of Langmuir isotherm used in the simulation software can be obtained after deformation:

$$
Q_{i}=\frac{I P_{1 i} I P_{2 i} c_{i}}{1+\sum_{k}\left(I P_{2 k} c_{k}\right)},
$$

where $\mathrm{IP}_{1 i}$ and $\mathrm{IP}_{2 i}$ are the adsorption parameters of component $i, c_{i}$ is the concentration of component $i$, and $Q_{i}$ is the adsorption amount of component $i$.

Linear driving force (LDF) was adopted in the simulation process, and Yang [18] approximately estimated the total mass transfer coefficient in the simulation:

$$
M T C_{s, i}=\frac{\Omega D_{e i}}{r_{p}^{2}}
$$

where $\Omega=15$. The research of Nakao and Suzuki [19] has shown that the $\Omega$ value is slightly higher than 15 when the adsorption balance in a short period of time. Assuming that the adsorption tower is in the intermediate time point in the cycle, we can calculate $\Omega$ through the following time constant:

$$
\theta=0.5 \frac{D_{e}}{r_{p}^{2}} t_{\text {Cycle }}
$$

$\Omega$ is a function of $\theta$ :

$\theta \geq 0.1: \Omega=15$,

$0.001 \leq \theta<0.1: \Omega(5.14 / \sqrt{\theta})$,

$\theta \leq 0.001: \Omega=162.5$.

The parameters of the feed gas are presented in Table 2, and the main parameters of the adsorption tower are shown in Table 3. During the simulation process design, the interactive module was used for the simulation of the double tower, and the four steps of intake, adsorption, depressurization, and desorption of PSA were controlled by the cycle controller.

The module was appropriately selected, and the whole process was controlled by the cycle controller. The process simulation diagram is shown in Figure 5.

\section{Results and Discussion}

The main system conditions that affect the separation effect of mine gas with low concentration by PSA are as follows: the properties of adsorbent, adsorption pressure, adsorption time, desorption time, feed gas concentration, adsorption tower structure, and other process parameters. PSA separation of low-concentration mine gas is achieved by pressure difference and selective adsorption of methane by adsorbent. Different adsorption times and desorption times are directly related to the integrity of the adsorption process. Pressure difference and time are particularly important for the PSA 
TABLE 1: Characteristic parameter of activated carbon.

\begin{tabular}{lccccc}
\hline $\begin{array}{l}\text { Specific surface } \\
\text { area }\left(\mathrm{m}^{2} \cdot \mathrm{g}^{-1}\right)\end{array}$ & $\begin{array}{c}\text { Pore volume of } \\
\text { adsorption }\left(\mathrm{cm}^{3} \cdot \mathrm{g}^{-1}\right)\end{array}$ & $\begin{array}{c}\text { Adsorption pore } \\
\text { diameter }(\mathrm{nm})\end{array}$ & $\begin{array}{c}\text { Pore volume of } \\
\text { desorption }\left(\mathrm{cm}^{3} \cdot \mathrm{g}^{-1}\right)\end{array}$ & $\begin{array}{c}\text { Desorption pore } \\
\text { diameter }(\mathrm{nm})\end{array}$ & $\begin{array}{c}\text { Saturated adsorption } \\
\text { capacity }\left(\mathrm{mL} \cdot \mathrm{g}^{-1}\right)\end{array}$ \\
\hline 1124.3 & $0.60806 D=799.34$ & 2.16327 & $0.53651 D=799.34$ & 1.90872 & 6.25 \\
\hline
\end{tabular}

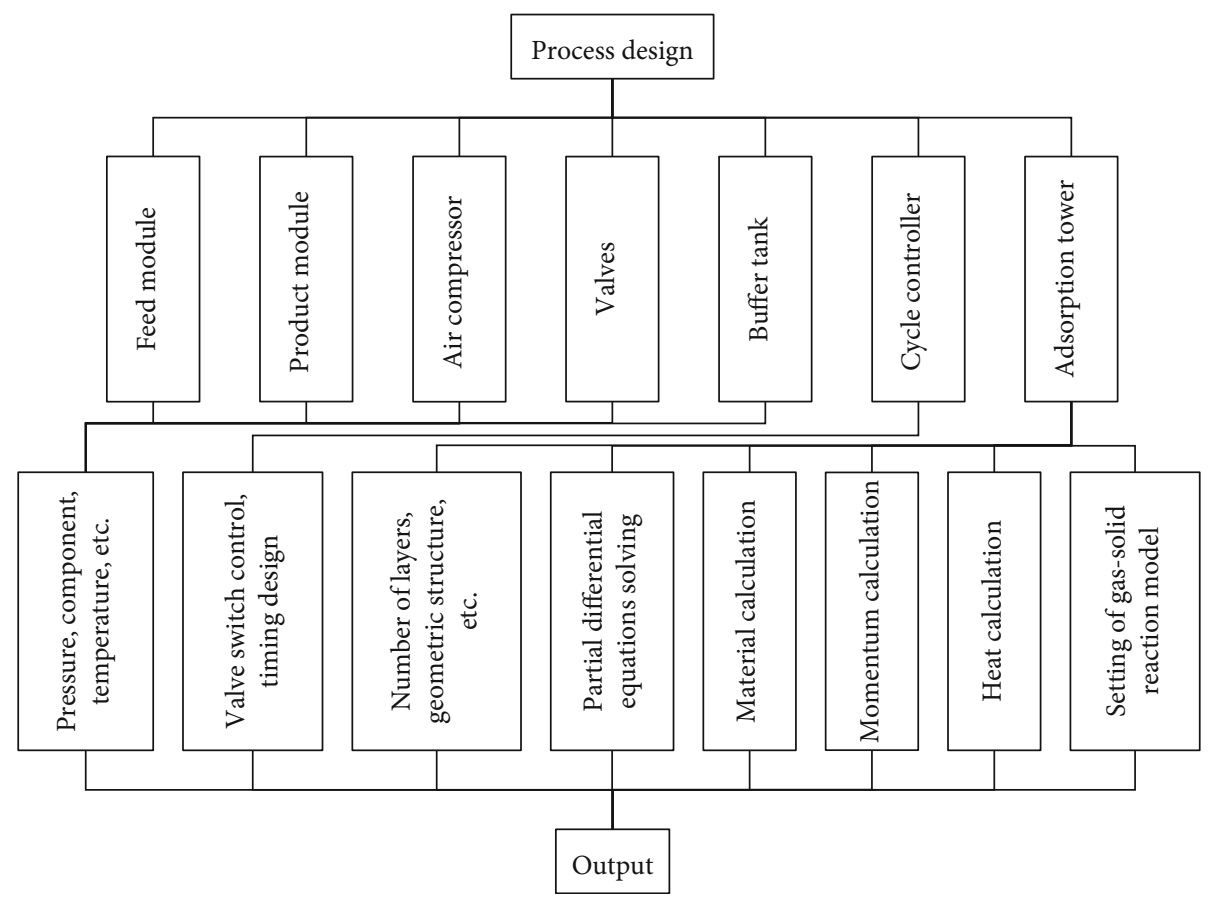

FIgURE 4: The schematic of Aspen Adsorption PSA simulation module.

TABLE 2: Feed gas parameters.

\begin{tabular}{lccc}
\hline Parameter & Value & Unit & Description \\
\hline$F$ & $1.34 e-005$ & $\mathrm{kmol} / \mathrm{s}$ & Flow rate \\
Y_Fwd (" $\mathrm{CH}_{4}$ ") & 0.003 & $\mathrm{kmol} / \mathrm{kmol}$ & Composition in forward direction \\
Y_Fwd ("N $\mathrm{N}_{2}$ ) & 0.997 & $\mathrm{kmol} / \mathrm{kmol}$ & Composition in forward direction \\
T_Fwd & 298.15 & $K$ & Temperature in forward direction \\
$P$ & 2.5 & Bar & Boundary pressure \\
\hline
\end{tabular}

TABLE 3: Adsorption tower parameters.

\begin{tabular}{lccc}
\hline Parameter & Value & Unit & Description \\
\hline $\mathrm{Hb}$ & 500 & $\mathrm{~mm}$ & Height of adsorbent layer \\
$\mathrm{Db}$ & 60 & $\mathrm{~mm}$ & Internal diameter adsorbent layer \\
$\mathrm{Ei}$ & 0.38 & $\mathrm{~m}^{3} \mathrm{void} / \mathrm{m}^{3}$ bed & Interparticle voidage \\
$\mathrm{Ep}$ & 0.7 & $\mathrm{~m}^{3} \mathrm{void} / \mathrm{m}^{3} \mathrm{bed}$ & Intraparticle voidage \\
$\mathrm{RHOs}$ & 512 & $\mathrm{~kg} / \mathrm{m}^{3}$ & Bulk solid density of adsorbent \\
$\mathrm{Rp}$ & 0.0012 & $\mathrm{~m}$ & Adsorbent particle radius \\
$\mathrm{Sfac}$ & 0.83 & $\mathrm{n} / \mathrm{a}$ & Adsorbent shape factor \\
MTC (“ $\left.\mathrm{CH}_{4}{ }^{\prime}\right)$ & 0.049 & $1 / \mathrm{s}$ & Constant mass transfer coefficients \\
MTC (“ $\mathrm{N}_{2}$ ”) & 0.092 & $1 / \mathrm{s}$ & Constant mass transfer coefficients \\
\hline
\end{tabular}




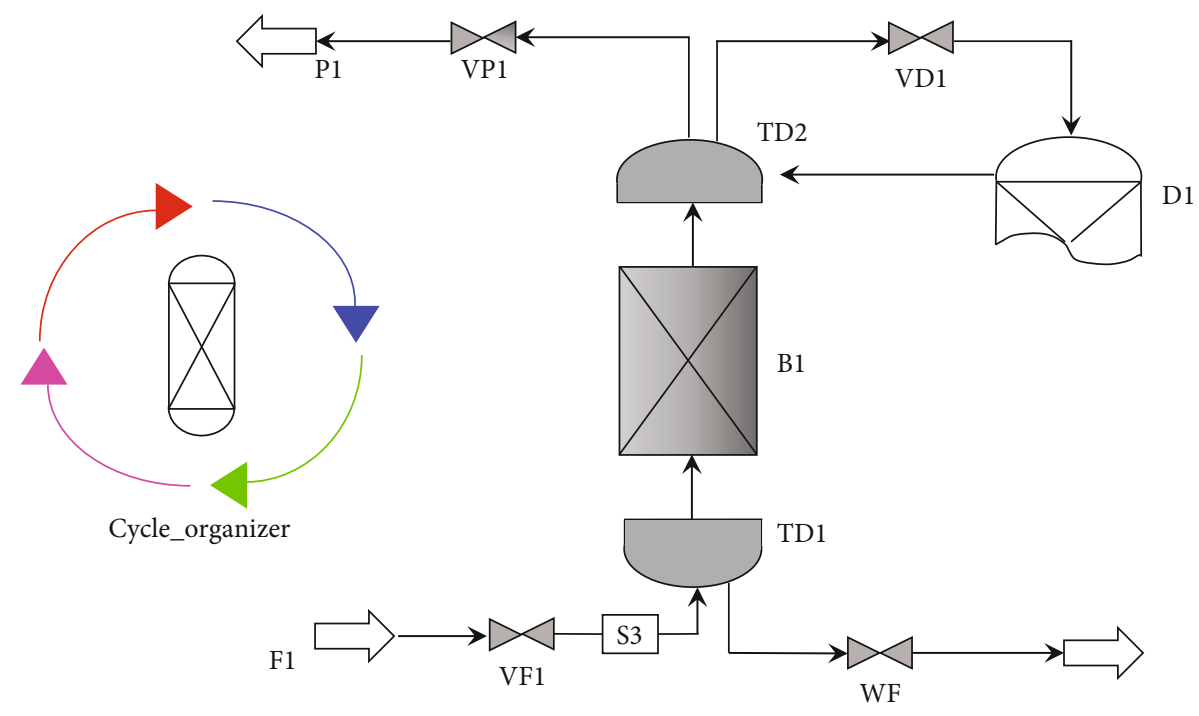

Figure 5: The process simulation diagram.

process. In addition, China's CVAM concentration is generally less than $0.75 \%$. Therefore, as with the experiment, four parameters including adsorption pressure, adsorption time, desorption time, and feed gas concentration were selected for simulation.

In the simulation process, the relevant parameters of adsorbent and experimental device were fully adopted, and parameters such as flow and flow rate were strictly input into the simulation software according to the readings of the display instrument in the experiment. In the experiment, the separation coefficients of nitrogen and oxygen were basically the same. In the simulation of adsorption separation, only nitrogen and methane were designed in the feed gas according to the treatment method of the experiment. After setting each parameter, the models would be initialized before each run to ensure the smooth progress of simulation.

\subsection{Effect of the Feed Gas Concentration}

3.1.1. Simulation Results. The methane concentration and wind volume of CVAM are unstable. However, China's coal mine ventilation air methane concentration is basically below $0.75 \%$. Therefore, the concentration of feed gas was set as $0.1 \%, 0.3 \%, 0.5 \%$, and $0.7 \%$ in the simulation study of PSA separation of CVAM. The software was initialized after all parameters had been input into the model. Then, the software was run after successful initialization. Under other conditions being the same, different methane concentrations of the feed gas were set to simulate the PSA process, and the variation curves of the desorbed methane concentration were obtained under different methane concentrations of feed gas, as shown in Figure 6.

It can be seen from Figure 6 that the change trend of each curve is consistent. At the beginning of desorption, the concentration of methane changes slowly, then gradually speeds up, and finally increases slowly until reaching the maximum desorption concentration. When the concentration of the feed gas was set at $0.1 \%$, methane concentration was increased to $0.21 \%$ after about 90 s desorption time, with an increase ratio of 2.1. When the concentration of the feed gas was $0.3 \%$, methane concentration was increased to $0.59 \%$ through $100 \mathrm{~s}$, with an increase ratio of 1.97 . When the concentration of the feed gas was $0.5 \%$, after desorption of $115 \mathrm{~s}$, the methane concentration was increased to $0.89 \%$, with an increase ratio of 1.78 . When the concentration of the feed gas was $0.7 \%$, after desorption time of $115 \mathrm{~s}$, the methane concentration was increased to $1.2 \%$, with an increase ratio of 1.71 . From the perspective of the curve, the optimal desorption time was $90 \mathrm{~s}$ to $115 \mathrm{~s}$ as the feed gas concentration increased from $0.1 \%$ to $0.5 \%$. Nevertheless, the optimal desorption time did not continue to increase with the increase of the feed gas concentration, and the concentration increase ratio was reduced by $18.6 \%$ from 2.1 to 1.71 . Within a certain range, the optimal desorption time tended to increase slowly with the increase of the feed gas concentration, while the increase ratio decreased slowly with the increase of the feed gas concentration.

3.1.2. Experimental Verification. Based on the current situation of ventilation air methane concentration in China's coal mines, feed gas concentrations of $0.1 \%, 0.3 \%, 0.5 \%$, and $0.7 \%$ were set to explore the influence of feed gas concentration on methane desorption concentration. Figure 7 shows the comparison between the experimental and simulation results of the effect of the feed gas concentration on the methane increase ratio. As shown in Figure 7, the simulation results are basically consistent with the experimental results; that is, with the increase of methane concentration of feed gas, the increase ratio of methane decreases instead. This can also be verified in the literatures of Sivakumar and Rao [20] and Dantas et al. [21]. For the three adsorption components of methane, nitrogen, and oxygen, the rangeability of methane adsorption amount is the largest at different partial pressures. Therefore, under the same conditions, the increase ratio of feed gas with a lower methane volume fraction is higher. As 


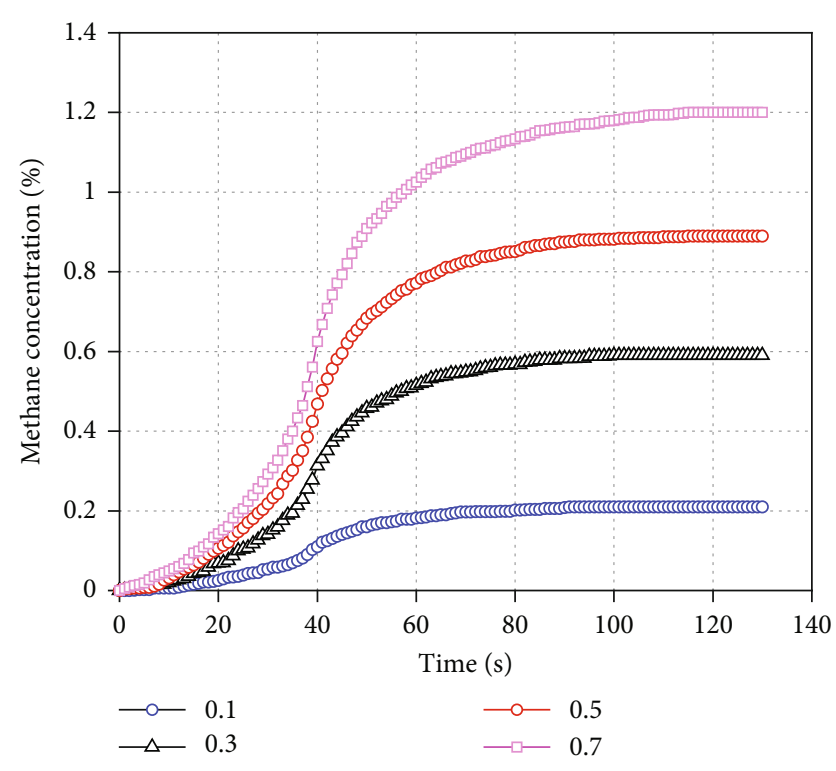

FIGURE 6: Influence of feed gas concentration on desorbed methane concentration.

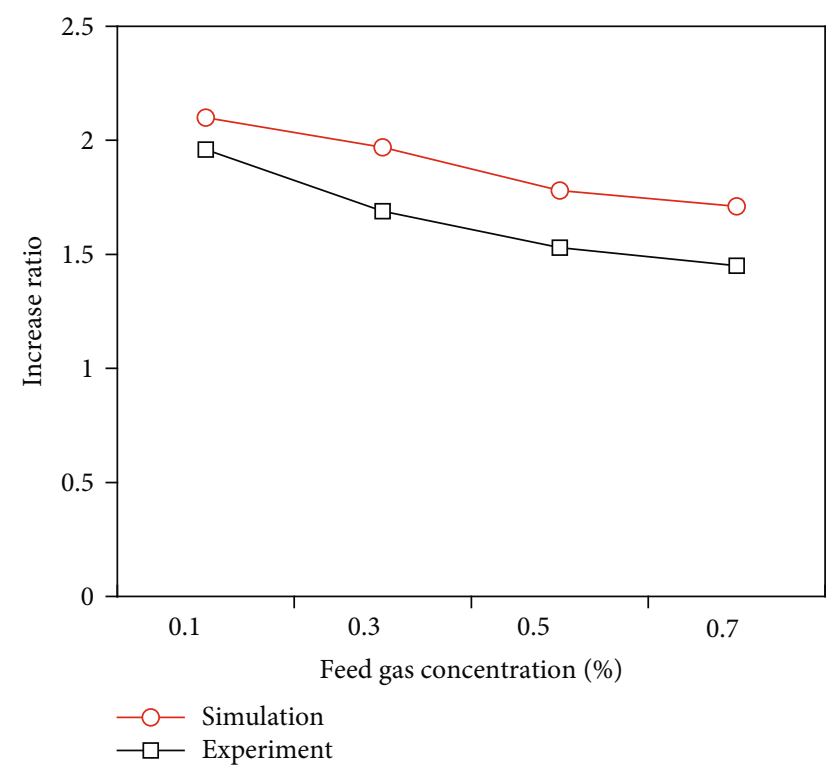

Figure 7: Comparison diagram of experiment and simulation.

the volume fraction of feed gas methane continued to increase, the increase ratio tended to be flat.

\subsection{Effect of the Adsorption Pressure}

3.2.1. Simulation Results. In the experiment of pressure swing adsorption, the pressure difference between adsorption and desorption is the key factor affecting the pressure swing adsorption concentrating methane of CVAM, and the concentrated methane concentration is generally proportional to the pressure difference. Because the desorption pressure in this experiment was constant at $55 \mathrm{kPa}$, the pressure difference was mainly adjusted by the change of adsorption pressure. Figure 8 shows the simulation result of the concentration of methane in product gas changes at the adsorption pressures of $120 \mathrm{kPa}, 150 \mathrm{kPa}, 180 \mathrm{kPa}, 210 \mathrm{kPa}$, and $240 \mathrm{kPa}$, respectively. When the adsorption pressure increased from $120 \mathrm{kPa}$ to $240 \mathrm{kPa}$ and the adsorbent loaded in the adsorption tower was only $40 \mathrm{~g}$, the inflection point at the bottom of the penetration curve changed from $70 \mathrm{~s}$ to $110 \mathrm{~s}$, and the penetration time increased by nearly $40 \mathrm{~s}$. The extension of penetration time can reduce the regeneration frequency of activated carbon and extend the service life of the adsorbent. Therefore, increasing adsorption pressure is good for methane adsorption, but increasing pressure requires more energy. The variation trend of curves under different pressures was roughly the same. The adsorption saturation point of the curves moved back with the adsorption pressure increased, but the basic shape of the curves remained the same. In the other words, the increase of adsorption pressure improved the adsorption capacity of the adsorption tower, and more methane gas could be absorbed in each cycle. Therefore, as the adsorption pressure increased, the desorption methane concentration increased.

3.2.2. Experimental Verification. Five gradients of $120 \mathrm{kPa}$, $150 \mathrm{kPa}, 180 \mathrm{kPa}, 210 \mathrm{kPa}$, and $240 \mathrm{kPa}$ were set in the experiment and simulation to investigate the effect of adsorption pressure on desorption methane concentration. Figure 9 shows the comparison between the experimental and simulation results of the effect of the adsorption pressure on the concentration of desorption methane. It can be seen from Figure 9 that the concentration of desorption methane increases with the increase of adsorption pressure, which is consistent with the results of studies in literature Anna et al. [22]. When the adsorption pressure was $210 \mathrm{kPa}$, the desorption methane concentration was the highest. However, when the adsorption pressure continued to rise to $240 \mathrm{kPa}$, the experimental results showed that the methane concentration was lower than that at $210 \mathrm{kPa}$, while the simulation results were the same as those at $210 \mathrm{kPa}$. There was a certain deviation between the simulation and the experimental results. In the actual operation, when the adsorption pressure exceeded a certain range, the mixed gas in the tower had already flowed out of the tower before fully contacting with the absorbent. The adsorption was not complete in the tower, and the amount of desorption methane would be reduced. However, this practical problem was not considered in the software simulation. Therefore, when using software simulation, it is not only necessary to strictly follow the relevant steps of the experiment but also to consider the deviation of the experiment and simulation results and find ways to improve the experiment and simulation process.

\subsection{Effect of the Adsorption Time}

3.3.1. Simulation Results. The adsorption time is the key parameter in the process of enrichment and separation of ventilation air methane by pressure swing adsorption, which directly affects the operation of enrichment and separation 


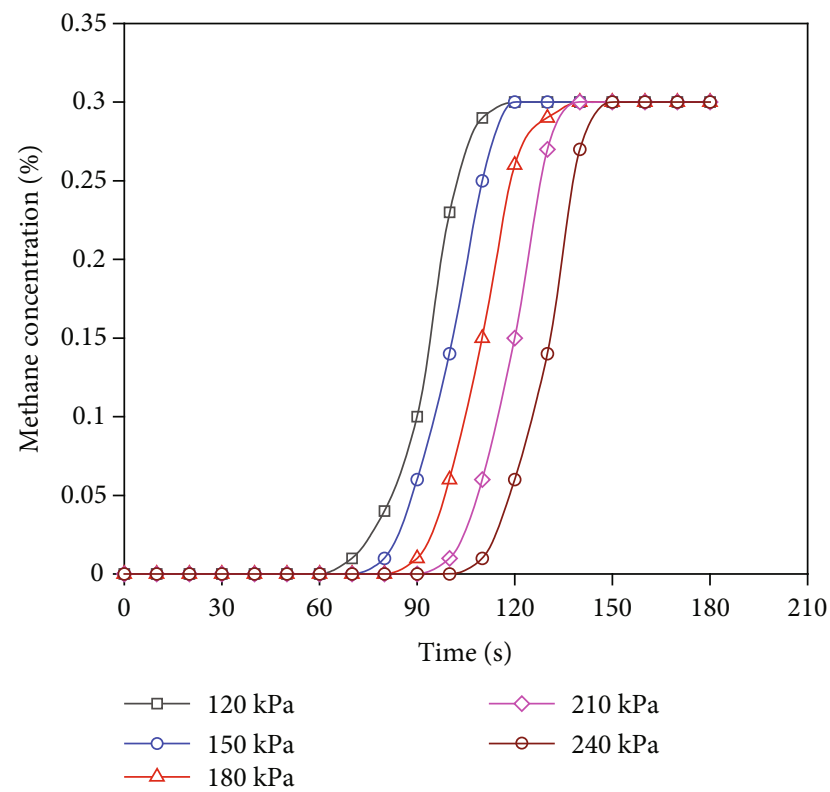

FIGURE 8: Influence of adsorption pressure on emission methane concentration.

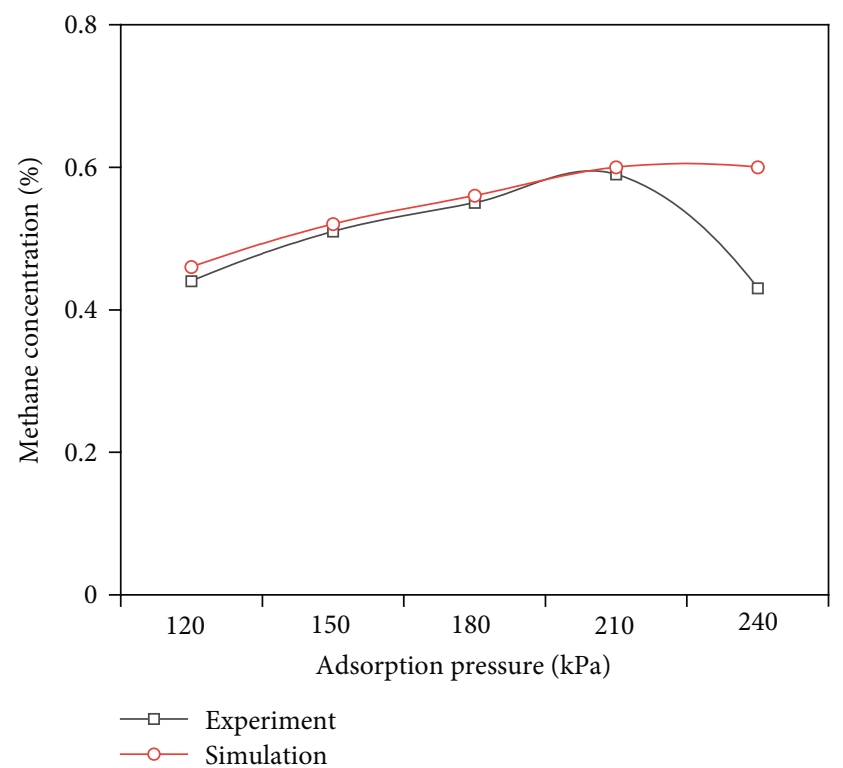

FIgURE 9: Comparison diagram of experiment and simulation.

system. The volume fraction of methane in desorption gas increases with the increase of adsorption time, but at the same time, the volume fraction of methane in effluent gas also increases. Proper adsorption time is very important for enrichment and separation of CVAM. In the cycle of vacuum pressure swing adsorption of the two towers, the two towers continuously switch between adsorption and desorption, and the adsorption time is controlled by switching the two towers.

Figure 10 shows the influence of simulated adsorption time on desorption methane concentration. As for the parameters selected in the experiment, four adsorption times, including $60 \mathrm{~s}, 90 \mathrm{~s}, 120 \mathrm{~s}$, and $150 \mathrm{~s}$ were set in the simulation

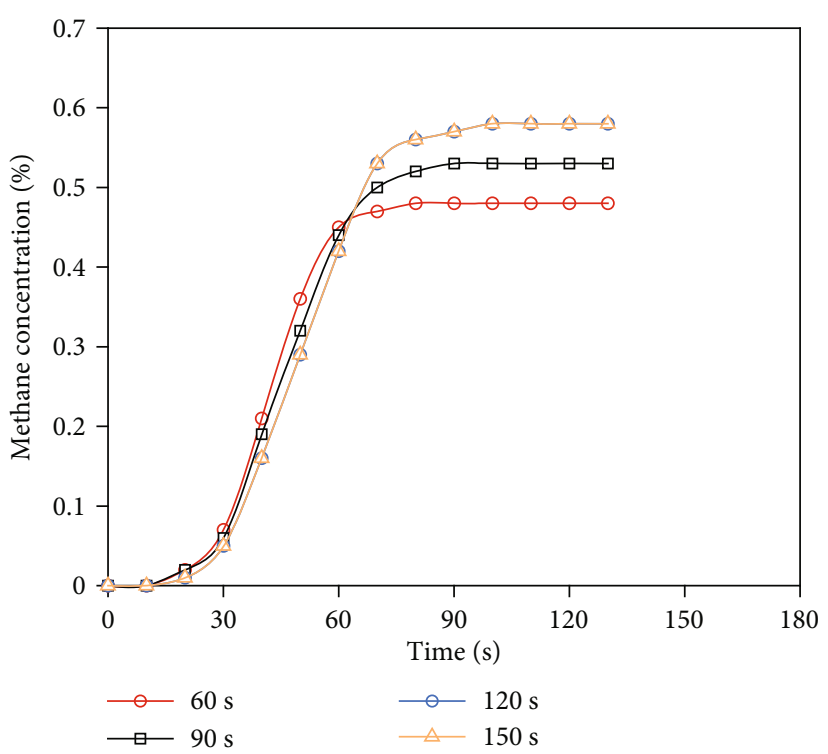

FIGURE 10: Influence of adsorption time on desorption methane concentration.

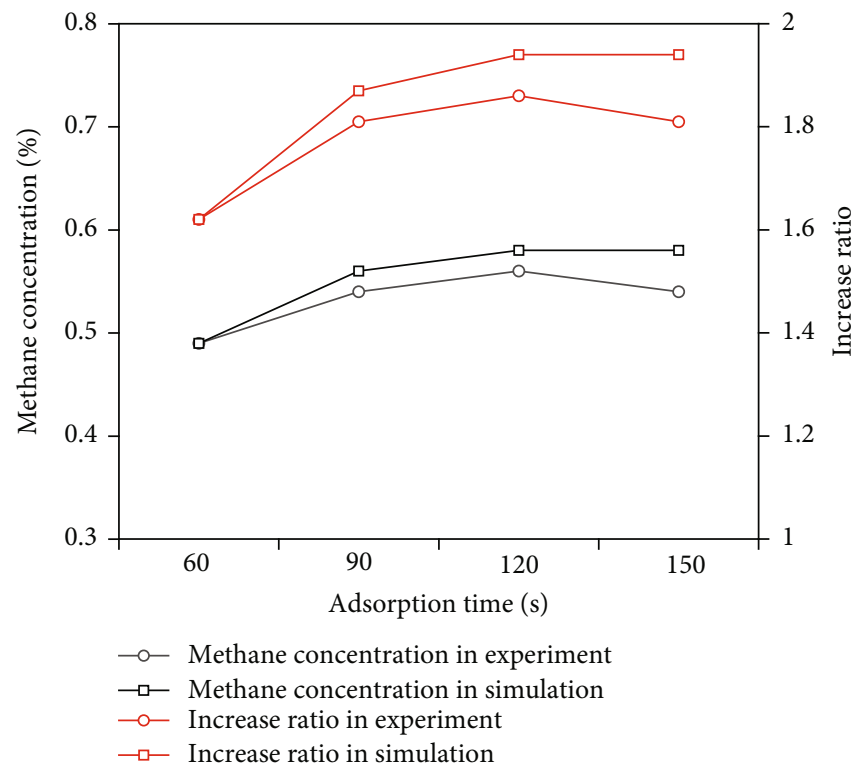

FIGURE 11: Experiment and simulation comparison chart.

to investigate the influence of different adsorption time on the desorption methane concentration. As can be seen from Figure 10, the four curves show the same change trend. At the beginning of desorption, methane concentration increased slowly and then rapidly increased to the maximum desorption concentration. Although the change trend was the same, when the adsorption time was $60 \mathrm{~s}$, the methane concentration was about $0.48 \%$; when adsorption time was $120 \mathrm{~s}$ and $150 \mathrm{~s}$, the methane concentration was nearly the same, about $0.58 \%$, which increased by $33 \%$ when the adsorption time was $60 \mathrm{~s}$. According to the simulation results, the methane concentration increased with the increase of adsorption time, and the optimal adsorption time was $120 \mathrm{~s}$. 


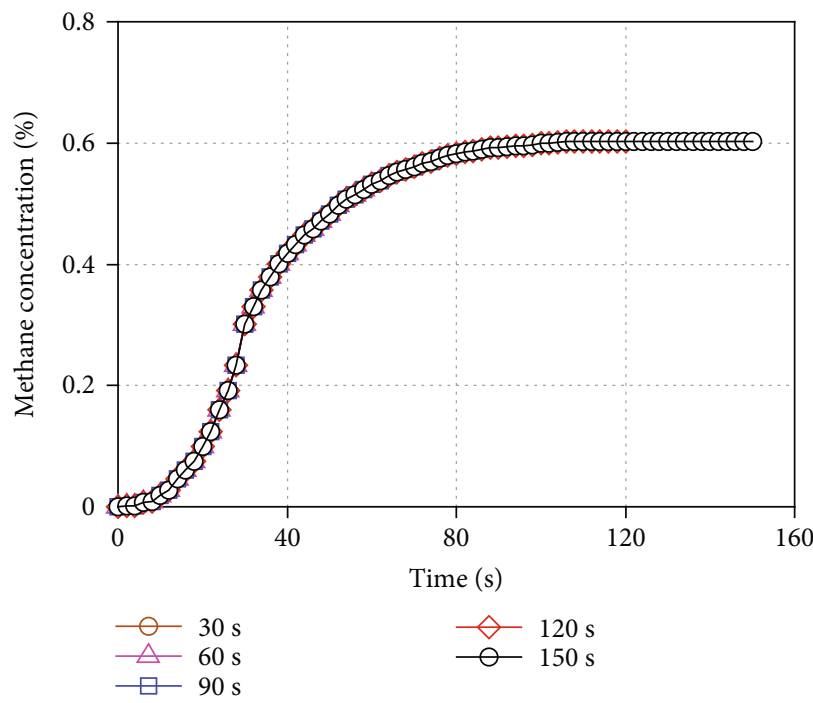

(a)

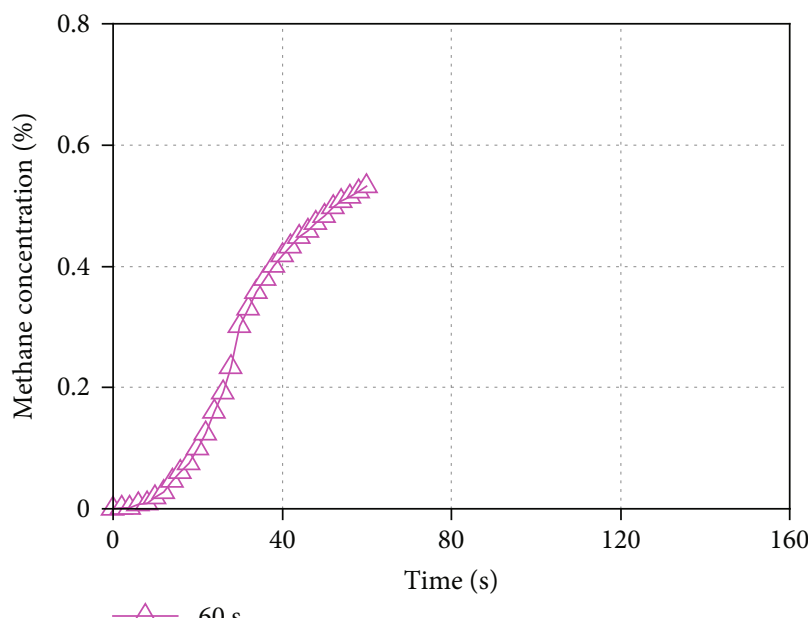

(c)

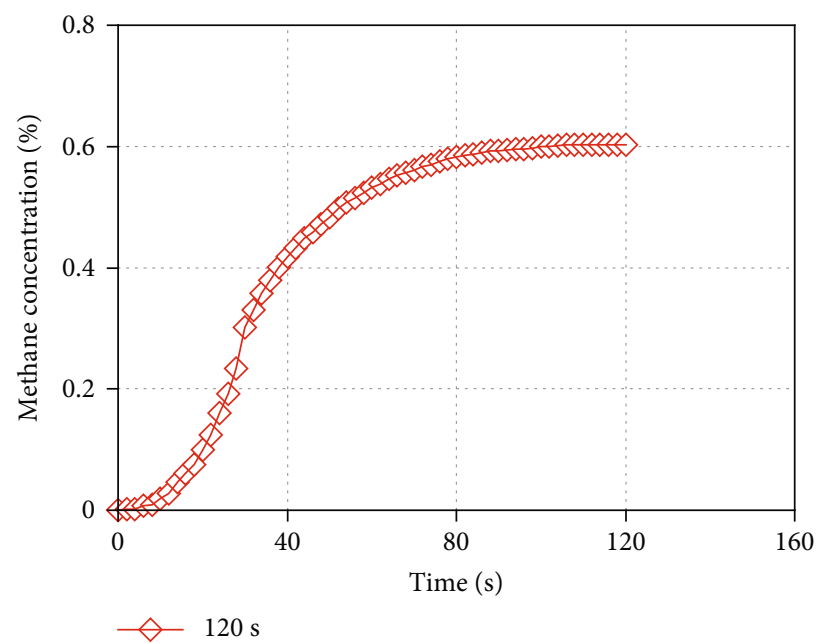

(e)

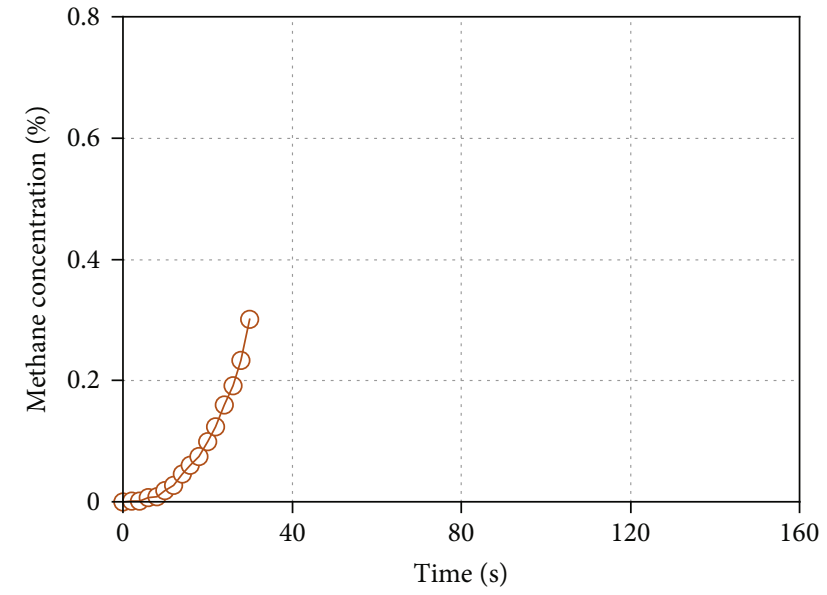

$-30 s$

(b)

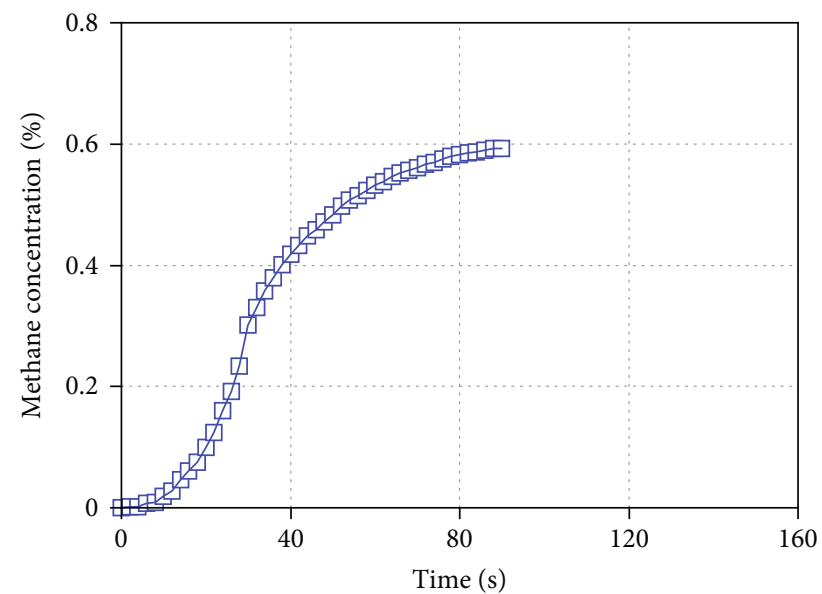

$\square-90 \mathrm{~s}$

(d)

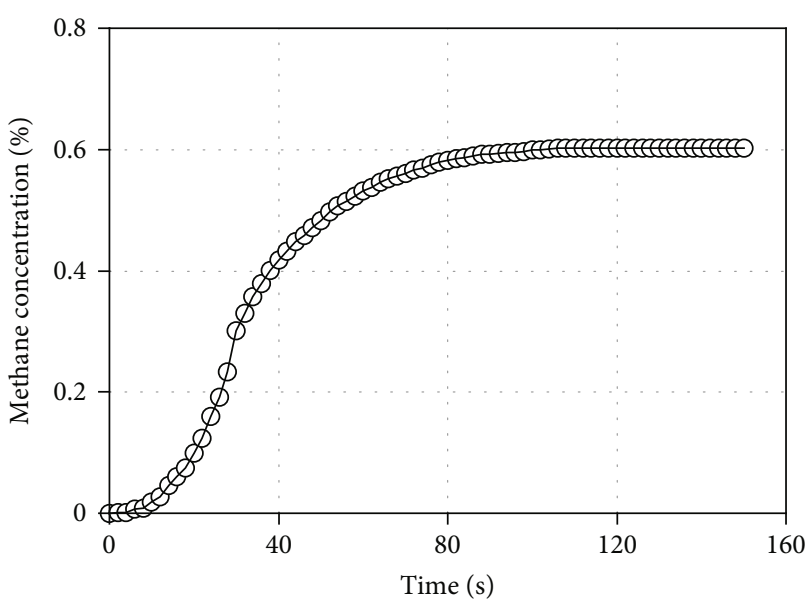

(f)

FIGURE 12: Influence of desorption time on desorption methane concentration. 
3.3.2. Experimental Verification. The adsorption time of $60 \mathrm{~s}$, $90 \mathrm{~s}, 120 \mathrm{~s}$, and $150 \mathrm{~s}$ was set in both experiment and simulation to verify the optimal adsorption time. Figure 11 shows the comparison of desorption methane concentration and increases ratio between experiment and simulation at different adsorption times. In the experiment, as the adsorption time increased, both the desorption methane concentration and increase ratio increased in a positive correlation. After reaching the optimal adsorption time of $120 \mathrm{~s}$, the methane concentration and the increase ratio showed a slight downward trend when the adsorption time continued to increase. This is because $120 \mathrm{~s}$ is the adsorption saturation time of device. Before that, the adsorption does not reach saturation, and more methane will be adsorbed with the time increasing, thus increasing desorption methane concentration. After reaching saturation, the amount of methane adsorbed by activated carbon will not increase.

According to Figure 11, the simulation results show that the optimal adsorption time is $120 \mathrm{~s}$. When the adsorption time exceeds the optimal adsorption time, the desorption methane concentration is almost consistent with that when the adsorption time is $120 \mathrm{~s}$. After many experiments and simulations, it was found that there was still deviation. It is concluded that the deviation may be due to the air tightness of the experimental device and resistance of the pipe and connection. Although the air tightness of the experimental device was checked with soapy water for each experiment to ensure the experiment is conducted under airtight conditions and this level of inspection fully met the accuracy required by the laboratory, the inspection tools like soapy water could only detect what the naked eye can see, and there was a certain gap compared with the software simulation. In addition, in the actual device, the pipelines were complicated and had many interfaces, which would affect the airflow, ${ }^{1}$ concentration, etc. The simulation software was a simplified model; so, there was an acceptable range of deviation between the experimental results and the simulation results.

\subsection{Effect of the Desorption Time}

3.4.1. Simulation Results. Whether desorption is complete depends on two points, one is desorption pressure difference, and the other is desorption time. The effect of desorption pressure difference on product gas methane concentration has been studied by changing the adsorption pressure. This section mainly discusses the effect of desorption time on product gas methane concentration. In practical production, if the desorption time is short, the desorption will not be complete, which will greatly reduce the concentration of methane in the product gas, and is not conducive to the regeneration of the adsorbent, which reduces the service life of the adsorbent. If the desorption time is long, it will increase the working pressure of the vacuum pump, easily damaging the vacuum pump, increasing energy consumption and operating cost. Therefore, the reasonable desorption time of the PSA system should be determined whether from the perspective of the service life of the equipment or from the perspective of energy saving and environmental protection.
The effect of desorption time on the concentration of desorption methane was simulated by setting the desorption time as $30 \mathrm{~s}, 60 \mathrm{~s}, 90 \mathrm{~s}, 120 \mathrm{~s}$, and $150 \mathrm{~s}$, respectively. It can be seen from Figure 12 that Figure 12(f) is a complete desorption curve, while Figures 12(b)-(e) are roughly an intercepted part of the figure (f) curve; that is, Figures 12(b)-(e) are incomplete desorption curves. Figure $12(\mathrm{a})$ is the integration of Figures 12(b)-(e). When the desorption time was $30 \mathrm{~s}$, there was basically no buffer in the simulation condition. Therefore, at the beginning of desorption, the methane concentration would rapidly rise to $0.301 \%$. When the desorption time was $90 \mathrm{~s}$, the increase ratio of desorption methane concentration began to slow down, and at this time, methane concentration was $0.583 \%$. The desorption time continued to increase, and methane concentration rose slowly. When the desorption time was $120 \mathrm{~s}$, desorption was basically complete, and methane concentration was $0.603 \%$. At this point, if the desorption time continued to increase, methane concentration would not change; so, the curve obtained with desorption time of $120 \mathrm{~s}$ was almost consistent with that obtained with desorption time of $150 \mathrm{~s}$. In conclusion, the optimal desorption time obtained by simulation was $120 \mathrm{~s}$.

3.4.2. Experimental Verification. Experimental and simulation results verify the variation trend of methane concentration when desorption time was $30 \mathrm{~s}, 60 \mathrm{~s}, 1990 \mathrm{~s}, 120 \mathrm{~s}$, and $150 \mathrm{~s}$. The optimal desorption time of simulation was $120 \mathrm{~s}$, while the result of the experiment was $150 \mathrm{~s}$. Although both methane concentrations rose to about $0.3 \%$ in desorption time $30 \mathrm{~s}$, the essence of the increase was different. In the experiment, the methane concentration rose to $0.38 \%$ when the desorption time was $30 \mathrm{~s}$. At this time, in order to avoid burning the vacuum pump, the adsorption tower was in the buffer stage, and the effluent gas was discharged from the adsorption tower to reduce the pressure in the tower. That is, desorption did not start in the adsorption tower. In the last cycle, residual methane residue was found in the methane detector and pipeline during desorption; so, the methane concentration was $0.38 \%$. In the simulation process, there was no burned out vacuum pump. Therefore, at the beginning of desorption, methane adsorbed on the adsorbent was desorbed. When the desorption time was $30 \mathrm{~s}$, the methane concentration detected was $0.301 \%$. Therefore, there was a 30 s time difference between the experimental results and the simulation results. In summary, the simulation results are consistent with the experimental results.

\section{Conclusions}

This paper mainly analyzes the simulation results of enrichment and separation of ventilation air methane from coal mine by pressure swing adsorption and experimentally verifies the effect of parameters such as feed gas concentration, adsorption pressure, adsorption time, and desorption time on the concentration of desorption methane. The results can be summarized as follows:

(1) In the simulation, the increase ratio of desorption methane decreases with the increase of methane 
concentration of feed gas, which is consistent with the experimental results. This is because methane has the largest adsorption range at different partial pressures among the three adsorption components of methane, nitrogen, and oxygen. Therefore, under the same conditions, the feed gas with a lower volume fraction of methane has a higher increase ratio. As the volume fraction of feed gas methane continues to increase, the increase ratio tends to be flat

(2) The simulation results are consistent with the experimental results: the optimal adsorption pressure is $210 \mathrm{kPa}$. However, when the adsorption pressure continued to rise to $240 \mathrm{kPa}$, the experimental results showed that the methane concentration was lower than that at $210 \mathrm{kPa}$, while the simulated result was the same as that at $210 \mathrm{kPa}$. The main reason for the difference is that the excessive pressure in the actual operation process leads to incomplete adsorption in the tower, the desorbed methane will be reduced, and the methane concentration will be reduced. The simulation process did not take this factor into account

(3) Both the simulation and experimental results indicate that the optimal adsorption time is $120 \mathrm{~s}$, and the concentration of desorption gas methane basically remains unchanged with the further adsorption time increase. Before the optimal adsorption time is reached, the adsorption did not reach saturation, and more methane will be absorbed with adsorption time increasing. After reaching saturation, the amount of methane adsorbed does not increase; so, the methane concentration does not increase

(4) Simulation results show that the best desorption time is $120 \mathrm{~s}$, while experimental results show that the best desorption time is $150 \mathrm{~s}$. The main reason for the difference is that, in order to avoid burning out the vacuum pump in the experimental process, there is an artificial buffer step for $30 \mathrm{~s}$ to reduce the pressure after the adsorption completing. Therefore, the experimental results have extended the optimal desorption time by $30 \mathrm{~s}$ compared with the simulation results

The comparison between experimental and simulation results shows that the experimental and simulation results are basically consistent. Therefore, Aspen Adsorption can be used in simulation study of enrichment and separation of CVAM by PSA. The study on the enrichment and separation of CVAM by PSA using Aspen Adsorption can reduce the investment cost and energy consumption and improve the efficiency of adsorption-separation and recovery, which is of great practical significance for the development of adsorption-separation and the protection of ecological environment.

\section{Data Availability}

This article is distributed under the terms of the Creative Commons Attribution 4.0 License (https:// creativecommons.org/licenses/by/4.0/) which permits any use, reproduction, and distribution of the work without further permission provided the original work is attributed as specified on the SAGE and Open Access pages (https://us .sagepub.com/en-us/nam/open-access-at-sage).

\section{Conflicts of Interest}

The author(s) declared no potential conflicts of interest with respect to the research, authorship, and/or publication of this article.

\section{Acknowledgments}

The authors also thank the editor and anonymous reviewers very much for their valuable advice. This study was supported by the Open Project Program of State Key Laboratory of Petroleum Pollution Control (Grant No. PPC2019013), CNPC Research Institute of Safety and Environmental Technology and Open Foundation of Shaanxi Key Laboratory of Lacustrine Shale Gas Accumulation and Exploitation (under planning), and the Fundamental Research Funds for the Central Universities (No. 2009QH03).

\section{References}

[1] Y. S. Liu, C. Z. Zhang, Y. L. Li, X. Yang, and Y. Meng, "Pressurization with raffinate in vacuum pressure-swing adsorption for upgrading ventilation air methane," Journal of China Coal Society, vol. 38, no. 11, pp. 1972-1976, 2013.

[2] Y. L. Li, C. Z. Zhang, Y. S. Liu, X. Yang, and Y. Meng, "Vacuum exhaust processes in vacuum pressure-swing adsorption for upgrading methane in ventilation air methane," Advanced Materials Research, vol. 479-481, pp. 260-265, 2012.

[3] X. Yang, Y. S. Liu, Y. L. Li, C. Z. Zhang, Y. Meng, and H. J. Yang, "Study on low concentration oxygen-bearing coal mine methane enrichment by pressure swing adsorption," Journal of China Coal Society, vol. 36, no. 1, pp. 91-96, 2011.

[4] T. Zhu, R. Chen, X. X. He et al., "Experimental study on separation and enrichment of ventilation air methane by vacuum pressure swing adsorption," Safety in Coal Mines, vol. 46, no. 9, pp. 1-4, 2015.

[5] R. Kumar, V. G. Fox, D. G. Hartzog et al., "A versatile process simulator for adsorptive separations," Chemical Engineering Science, vol. 49, no. 18, pp. 3115-3125, 1994.

[6] Y. Liu and J. A. Ritter, "Pressure swing adsorption-solvent vapor recovery: process dynamics and parametric study," Industrial \& Engineering Chemistry Research, vol. 35, no. 7, pp. 2299-2312, 1996.

[7] L. Jiang, L. T. Biegler, and V. Grant Fox, "Design and optimization of pressure swing adsorption systems with parallel implementation," Computer Aided Chemical Engineering, vol. 15, pp. 232-237, 2003.

[8] P. S. Bárcia, J. A. C. Silva, and A. E. Rodrigues, “Adsorption dynamics of $\mathrm{C}_{5}-\mathrm{C}_{6}$ Isomerate fractions in zeolite beta for the octane improvement of gasoline," Energy \& Fuels, vol. 24, no. 3, pp. 1931-1940, 2010.

[9] S. Yang, D. Choi, S. Jang, S. H. Kim, and D. K. Choi, "Hydrogen separation by multi-bed pressure swing adsorption of synthesis gas," Adsorption, vol. 14, no. 4-5, pp. 583-590, 2008. 
[10] Y. Ding and M. D. Levan, "Periodic states of adsorption cycles III. Convergence acceleration for direct determination," Chemical Engineering Science, vol. 56, no. 17, pp. 5217-5230, 2001.

[11] L. Jiang, L. T. Biegler, and V. G. Fox, "Simulation and optimization of pressure-swing adsorption systems for air separation,” AICHE Journal, vol. 49, no. 5, pp. 1140-1157, 2003.

[12] S. Nilchan and C. C. Pantelides, "On the optimisation of periodic adsorption processes,” Adsorption, vol. 4, no. 2, pp. 113-147, 1998.

[13] F. A. da Silva, J. A. Silva, and A. E. Rodrigues, "A general package for the simulation of cyclic adsorption processes," Adsorption, vol. 5, no. 3, article 231007, pp. 229-244, 1999.

[14] Y. Yang, Y. Wu, H. Liu et al., "Enrichment of ventilation air methane by adsorption with displacement chromatography technology: experiment and numerical simulation," Chemical Engineering Science, vol. 149, pp. 215-228, 2016.

[15] D. Qu, Y. Yang, K. Lu et al., "Microstructure effect of carbon materials on the low-concentration methane adsorption separation from its mixture with nitrogen," Adsorption, vol. 24, no. 4, pp. 357-369, 2018.

[16] “Adsorption Reference Guide-ASPEN ADSIMTM11.1," http://www.aspentech.com.

[17] D. Qu, Y. Yang, Z. Qian et al., "Enrichment of low-grade methane gas from nitrogen mixture by VPSA with $\mathrm{CO}_{2}$ displacement process: modeling and experiment," Chemical Engineering Journal, vol. 380, article 122509, 2020.

[18] R. T. Yang, Gas Separation by Adsorption Processes, Butterworth, Boston, 1986.

[19] S. Nakao and M. Suzuki, "Mass Transfer coefficient in cyclic adsorption and desorption," Journal of Chemical Engineering of Japan, vol. 16, no. 2, pp. 114-119, 1983.

[20] S. V. Sivakumar and D. P. Rao, "Modified duplex PSA.1. Sharp separation and process intensification for $\mathrm{CO}_{2}-\mathrm{N}_{2}-13 \mathrm{X}$ zeolite system," Industrial and Engineering Chemistry Research, vol. 50, no. 6, pp. 3426-3436, 2011.

[21] T. L. P. Dantas, F. M. T. Luna, I. J. Silva Jr. et al., "Carbon dioxide-nitrogen separation through pressure swing adsorption," Chemical Engineering Journal, vol. 172, no. 2-3, pp. 698-704, 2011.

[22] H. R. Sant Anna, A. G. Barreto Jr., F. W. Tavares, and J. F. do Nascimento, "Methane/nitrogen separation through pressure swing adsorption process from nitrogen-rich streams," Chemical Engineering and Processing: Process Intensification, vol. 103, pp. 70-79, 2016. 